\title{
Fauna de hidrozoários atecados (Hydrozoa, Anthoathecata) da costa do Estado do Ceará, Brasil
}

\author{
Vanessa Shimabukuro ${ }^{1}$, Antonio C. Marques $^{1}$ \& Alvaro E. Migotto ${ }^{1,2}$
}

Biota Neotropica v6 (n3) -http://www.biotaneotropica.org.br/v6n3/pt/abstract?inventory+bn00806032006

Recebido em 08/09/2005.

Versão reformulada recebida em 08/06/2006

Publicado em 01/09/2006

\footnotetext{
${ }^{1}$ Departamento de Zoologia, Instituto de Biociências, Universidade de São Paulo. São Paulo, SP, Brasil. [vshima@ib.usp.br; marques@ib.usp.br]

${ }^{2}$ Centro de Biologia Marinha, Universidade de São Paulo. Av. Manoel H. do Rego km 131,5; 11600-000 São Sebastião, SP, Brasil [aemigott@usp.br]
}

\begin{abstract}
Shimabukuro, V., Marques, A.C. and Migotto, A.E. The anthoathecate hydroid fauna (Hydrozoa, Anthoathecata) of the coast of Ceará State, Brazil. Biota Neotrop. Sep/Dec 2006 vol. 6, no. 3 http://www.biotaneotropica.org.br/v6n3/pt/ abstract?inventory+bn00806032006 ISSN 1676-0603
\end{abstract}

Species of athecate hydroids collected on beaches of the coast of Ceará State were studied. Nine species were found: Bimeria vestita, Pennaria disticha, Eudendrium caraiuru, Eudendrium carneum, Eudendrium pocaruquarum, Eudendrium sp., Sphaerocoryne sp., Porpita porpita and Coryne sp., all recorded for the first time in the region.

Key words: Hydrozoa, Anthoathecata, Capitata, Filifera.

\section{Resumo}

Shimabukuro, V., Marques, A.C. e Migotto, A.E. Fauna de hidrozoários atecados (Hydrozoa, Anthoathecata) da costa do Estado do Ceará, Brasil. Biota Neotrop. Sep/Dec 2006 vol. 6, no. 3 http://www.biotaneotropica.org.br/v6n3/pt/ abstract?inventory+bn00806032006 ISSN 1676-0603

Espécies de hidrozoários atecados coletadas em praias da costa do estado do Ceará foram estudadas. No total foram encontradas nove espécies: Bimeria vestita, Pennaria disticha, Eudendrium caraiuru, Eudendrium carneum, Eudendrium pocaruquarum, Eudendrium sp., Sphaerocoryne sp., Porpita porpita e Coryne sp., todas registradas pela primeira vez para a região.

Palavras-chave: Hydrozoa, Anthoathecata, Capitata, Filifera.

http://www.biotaneotropica.org.br 


\section{Introdução}

O litoral do Estado do Ceará tem aproximadamente $600 \mathrm{~km}$ de extensão, caracterizado por uma costa heterogênea, com praias arenosas ou constituídas por rochas, falésias, dunas, lagoas, rios, arrecifes e manguezais. A variedade de ambientes relacionados à zona costeira marinha propicia o desenvolvimento de uma fauna bastante diversificada, especialmente de animais sésseis, que encontram, em alguns desses ambientes, locais favoráveis para a fixação.

O estudo de hidrozoários no Brasil vem se intensificando nos últimos anos, em especial a partir de sua retomada na primeira metade do século, como os de Vannucci para algumas localidades da costa do Sul, Sudeste e Nordeste (Vannucci-Mendes 1946, Vannucci 1951a, 1951b, 1954). Dentre os trabalhos publicados, há estudos ecológicos (e.g. Rosso \& Marques 1997, Calder \& Maÿal 1998), incluindo abordagens relativas a impacto ambiental (Nogueira et al. 1997). No entanto, a maioria dos artigos sobre hidrozoários é constituída de levantamentos faunísticos de determinadas áreas da costa brasileira (e.g. Migotto 1996, Grohmann et al. 1997, Kelmo \& de SantaIsabel 1998), de grupos restritos tratados para regiões mais extensas (e.g. Marques 2001) ou de compilações para a costa como um todo (Migotto et al. 2002). Sob uma perspectiva geográfica e histórica, a região Sudeste tem recebido maior atenção, o Norte e o Nordeste estando entre as regiões menos estudadas (cf. Migotto et al. 2002, Marques et al. 2003).

Estão listadas 357 espécies de Hydrozoa para o Brasil, 96 das quais pertencentes à subclasse Anthoathecata (Migotto et al. 2002, Marques et al. 2003, Grohmann et al. 2003). Os atecados, como são coloquialmente conhecidos os membros dessa subclasse, são caracterizados por apresentar o esqueleto quitinoso restrito à hidrorriza e hidrocaule, mas sem a presença de uma hidroteca, estrutura característica dos Leptothecata (tecados) (Millard 1975). Outras características dos atecados são polimorfismo de pólipos, estrutura variada dos tentáculos da medusa e do pólipo, arranjados neste último em dois ou mais verticilos; na medusa, há gônadas no manúbrio ou canais radiais, presença de mesentérios perradiais e de ocelos (Marques $\&$ Collins 2004).

Um total de 36 espécies de atecados é reportado para a região Nordeste do Brasil, com os maiores números de registros para os estados da Bahia e Pernambuco, com 15 espécies cada (Migotto et al. 2002; Marques et al. 2003). O estado do Ceará conta com apenas uma espécie registrada na literatura, Millepora alcicornis Linnaeus, 1758 (cf. Migotto et al. 2002), o que demonstra um claro hiato de conhecimento sobre sua costa. Uma análise mais profunda dos estudos referentes à biodiversidade brasileira mostra uma grande carência de conhecimento (cf. Migotto \&
Marques 2006), inclusive sobre hidrozoários, para o Norte e parte do Nordeste. A situação é ainda mais crítica quando se considera ambientes marinhos de maiores profundidades (Marques et al. 2003; Migotto \& Marques 2006).

Este trabalho é parte de um projeto maior, o estudo da "Biota marinha da costa oeste do Ceará". Financiado pelo Ministério do Meio Ambiente (MMA), no âmbito do "Projeto de conservação e utilização sustentável da diversidade biológica brasileira" (Probio). O projeto objetiva a caracterização dos organismos bentônicos de substratos consolidados e não consolidados da área considerada como de alta prioridade pelo MMA, como resultado do Workshop "Avaliação de Ações Prioritárias para Zonas Costeira e Marinha”. Nas últimas décadas a área enfocada tem sofrido impactos oriundos de diversas atividades humanas, tais como turismo, especulação imobiliária, construção de um terminal marítimo e, mais recentemente, daqueles advindos da implantação do Porto do Pecém. O objetivo específico deste estudo foi realizar um levantamento faunístico e caracterizar as diferentes espécies de Hydrozoa atecados encontrados na costa do estado do Ceará.

\section{Material e métodos}

As coletas foram realizadas em diferentes pontos da costa do estado do Ceará (Figura 1), utilizando metodologias diversas, de coletas manuais a dragagens. A primeira coleta foi no período entre outubro e novembro de 2002, no município de Trairi ( $\left.39^{\circ} 16^{\prime} \mathrm{W}, 03^{\circ} 16^{\prime} \mathrm{S}\right)$, nas praias de Fleixeiras e Mundaú, e no município de Caucaia (38³9'W, 034' S), na praia do Pacheco. A segunda estação de coleta foi entre março e junho de 2003, no município de Trairi, praia de Guajiru e no município de Paracuru (39 $\left.01^{\circ} \mathrm{W}, 03^{\circ} 24^{\prime} \mathrm{S}\right)$, praia de Paracuru. A última coleta foi realizada no mês de outubro de 2003 no município de Pecém (3850'W, 03³3'S), praia de Pecém, no município de Cascavel (38 $14^{\circ}$ 'W, 04º7'S), praia de Caponga e no município de Paracuru, praia de Paracuru. Ademais, materiais de coleções prévias (indicados com data anterior às coletas) também foram estudados, estes provenientes dos municípios de Itapipoca (39 $49^{\circ} \mathrm{W}$, $\left.03^{\circ} 21^{\prime} \mathrm{S}\right)$, praia da Baleia; Fortaleza (38 $\left.32^{\prime} \mathrm{W}, 03^{\circ} 43^{\prime} \mathrm{S}\right)$, praias de Mucuripe e Sabiaguaba, além de outros materiais coletados ao largo da costa; e Cascavel, praia de Caponga.

As coletas realizadas nos anos de 2002 e 2003 foram manuais, sobre substrato consolidado em águas rasas (profundidade de zero a um metro). O material coletado foi anestesiado em solução de cloreto de magnésio a 7,5\% e fixado com solução de formol salino a $4 \%$ ou em álcool etílico a $70 \%$. O material foi triado sob lupa em laboratório. As coletas realizadas antes de 2002 utilizaram metodologias variadas, como dragagem e coletas em recifes artificiais, até uma profundidade de $15 \mathrm{~m}$, conforme indicado junto à lista de material estudado. 


$$
\mathbb{E}
$$




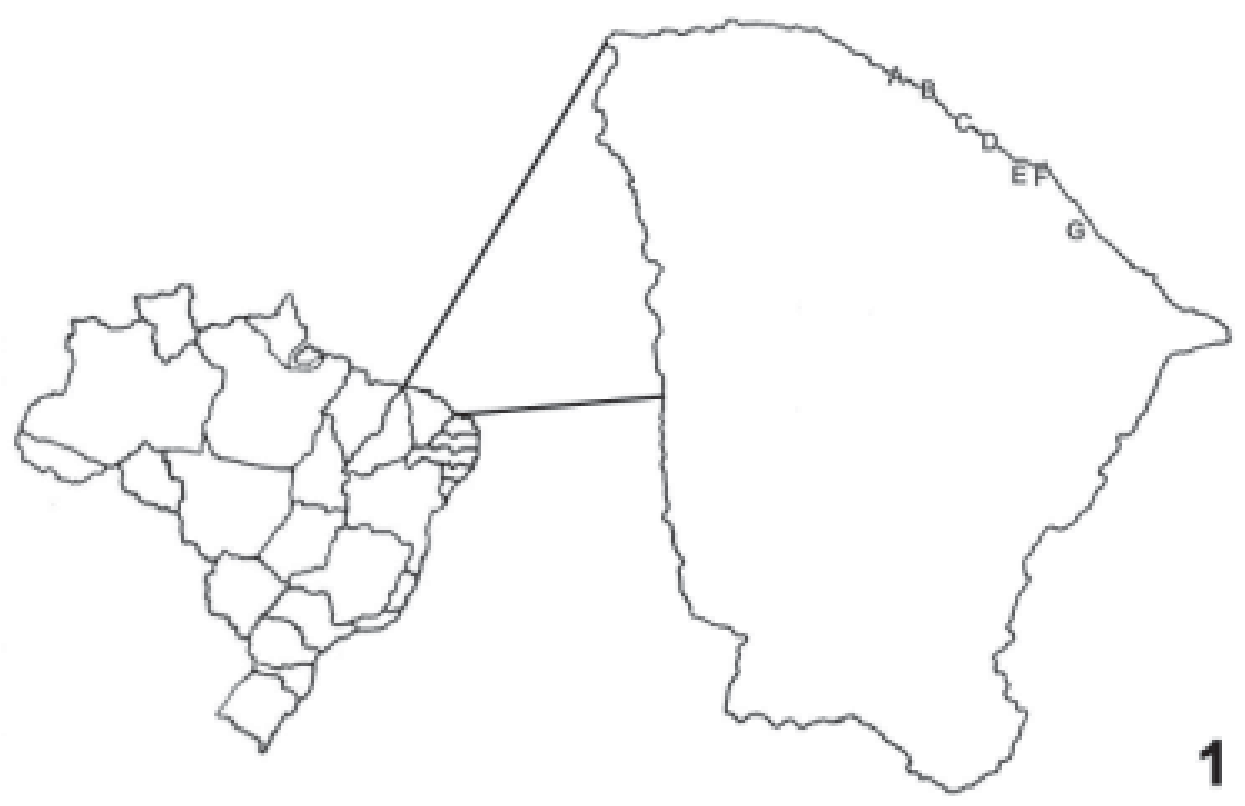

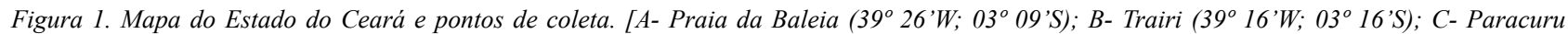

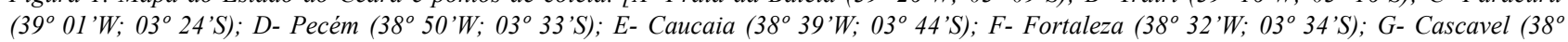
$\left.\left.14^{\prime} \mathrm{W} ; 04^{\circ} 07^{\prime} S\right)\right]$

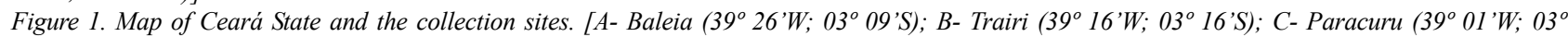

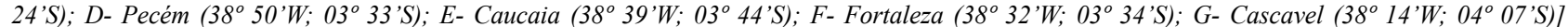

A análise do cnidoma, com medidas de nematocistos não explodidos, foi feita para a determinação das espécies de Eudendrium (cf. Marques 2001). A terminologia utilizada para o cnidoma foi baseada em Weill (1934), Mariscal (1974) e Östman (2000).

$\mathrm{Na}$ preparação dos materiais para microscopia eletrônica de varredura, fragmentos de colônias foram pósfixados em tetróxido de ósmio $\left(\mathrm{OsO}_{4}\right)$ a $1 \%$, desidratados em série de etanol em diferentes concentrações, secos ao ponto crítico e metalizados com ouro (Migotto \& Marques 1999).

Os materiais identificados foram incorporados às coleções científicas do Laboratório de Malacologia e Invertebrados Marinhos do Departamento de Biologia e do Instituto de Ciências do Mar da Universidade Federal do Ceará e na coleção de Cnidários do Museu de Zoologia da Universidade de São Paulo.

\section{Resultados e Discussão}

Foram encontradas nove espécies de hidrozoários atecados, pertencendo a seis gêneros e seis famílias distintas: Bimeria vestita Wright, 1859; Pennaria disticha
Goldfuss, 1820; Eudendrium caraiuru Marques \& Oliveira, 2003; Eudendrium carneum Clarke, 1882; Eudendrium pocaruquarum Marques, 1995; Eudendrium sp., Coryne sp., Sphaerocoryne sp. e Porpita porpita (Linnaeus, 1758). As espécies foram listadas abaixo, incluindo descrições e figuras. Na tabela I está a síntese das ocorrências das espécies nos locais de coleta.

\section{Classe Hydrozoa Huxley, 1856 \\ Subclasse Anthoathecata Cornelius, 1992 \\ Ordem Filifera Kühn, 1913}

Família Bougainvilliidae Lütken, 1850

Bimeria vestita Wright, 1859

Bimeria vestita Wright 1859: 109; Calder 1988: 21-23, figs. 17-18a-b; Millard 1975: 95, fig. 32C-H; Migotto 1996: 9 , fig. 2a-b; Grohmann et al. 1997: 230; Rosso \& Marques 1997: 417; Kelmo \& de Santa-Isabel 1998: 63, 65-66, fig. 6; Marques et al. 2000a: 321-325, figs. 1-3; Migotto et al. 2002: 11.

[Sinonímia completa em Calder 1988 e Marques et al. 2000a].

http://www.biotaneotropica.org.br 
Material.- Itapipoca: praia da Baleia, colônias com gonóforo 02.iv.1996, 15 m, sobre E. carneum, col. W. Franklin Jr., álcool (MZUSP 522); Trairi: praia de Fleixeiras, quatro amostras, colônias com gonóforos, 03.xi.2002, entremarés, col. A.C. Marques, álcool (MZUSP 506, 514, 516 e 521); Paracuru: praia de Paracuru, três amostras, colônias com e sem gonóforo, 27.x.2003, entremarés, col. A.C. Marques \& V. Shimabukuro, formol (MZUSP 646, 650 e 654); Paracuru: praia de Paracuru, colônias sem gonóforo, 16.vi.2003, entremarés, col. indeterminado, álcool (MZUSP 662); Cascavel: praia de Caponga, onze amostras, colônias com e sem gonóforo, 26.x.2003, entremarés, col. A.C. Marques \& V. Shimabukuro, formol (MZUSP 605, 607, 611, 613, 616, 620, $622,624,632,639$ e 645$)$.

Descrição.- Colônia ereta, pouco ramificada alternadamente, ou com pedículos surgindo diretamente de hidrorriza rastejante pouco ramificada; perissarco do hidrocaule afasciculado (diâmetro 74-96 $\mu \mathrm{m}$ ) e pedículos

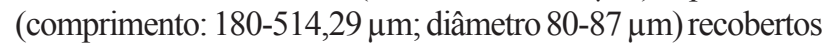
por detritos, com anelações ou rugas esparsas. Hidrante

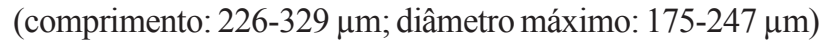
com hipostômio cônico, circundado por tentáculos filiformes (12-14; comprimento: 105,41-195,74 $\mu \mathrm{m}$ ) arranjados em dois verticilos próximos; pseudohidroteca presente, envolvendo a parte basal dos tentáculos.

Gonóforos ovóides ou esféricos (diâmetro: 158,35-

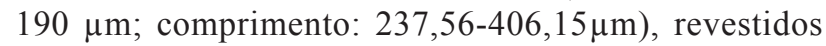
completamente por quitina e detritos, com pedículos finos e curtos, surgindo dos hidrocaules abaixo do hidrante.

Referências para o Brasil._- Migotto 1996: 9, fig. 2ab; Grohmann et al. 1997: 230; Rosso \& Marques 1997: 417; Kelmo \& de Santa-Isabel 1998: 63, 65-66, fig. 6; Migotto et al. 2002: 11; Grohmann et al. 2003: 18; Marques \& Migotto 2004: 174.

Ocorrências no Ceará.- Jericoacoara, praia de Jericoacoara; Itapipoca, praia da Baleia; Trairi, praias de Mundaú, Fleixeiras e Guajiru; Paracuru, praia de Paracuru; Taíba, praia da Taíba; Caucaia, praia do Pacheco; Cascavel, praia de Caponga. Novo registro para o Ceará.

Distribuição.- Brasil: São Paulo, Espírito Santo, Bahia, Pernambuco. Geral: cosmopolita (oceanos Atlântico, Índico e Pacífico).

Comentários. - O material cearense é semelhante ao material de São Sebastião, SP (Migotto 1996) em relação ao número de tentáculos, tamanho dos gonóforos e diâmetro do hidrocaule. O comprimento dos tentáculos é menor no material nordestino, o que deve estar relacionado a condições de anestesia e preservação. Não há também diferenças evidentes entre o material cearense e aqueles reportados para outros locais do Atlântico, como o sul africano (Millard 1975) e bermudense (Calder 1988). A espécie estudada foi freqüentemente encontrada sobre outros hidrozoários e algas, desde a zona entremarés até $15 \mathrm{~m}$ de profundidade.

A espécie Bimeria vestita apresenta uma distribuição fragmentada entre as regiões Sudeste e Nordeste, embora conte com distribuição mundial ampla, sendo registrada até mesmo para águas sub-antárticas (Marques et al. 2000a). Por ser uma espécie relativamente pequena e comumente epizóica, sua distribuição e abundância podem estar subestimadas. As colônias são, geralmente, recobertas por matéria orgânica aglutinada e silte, e seus hidrantes tendem a ficar retraídos, o que dificulta sua caracterização e identificação.

Família Eudendriidae L. Agassiz, 1862

Eudendrium caraiuru Marques \& Oliveira, 2003

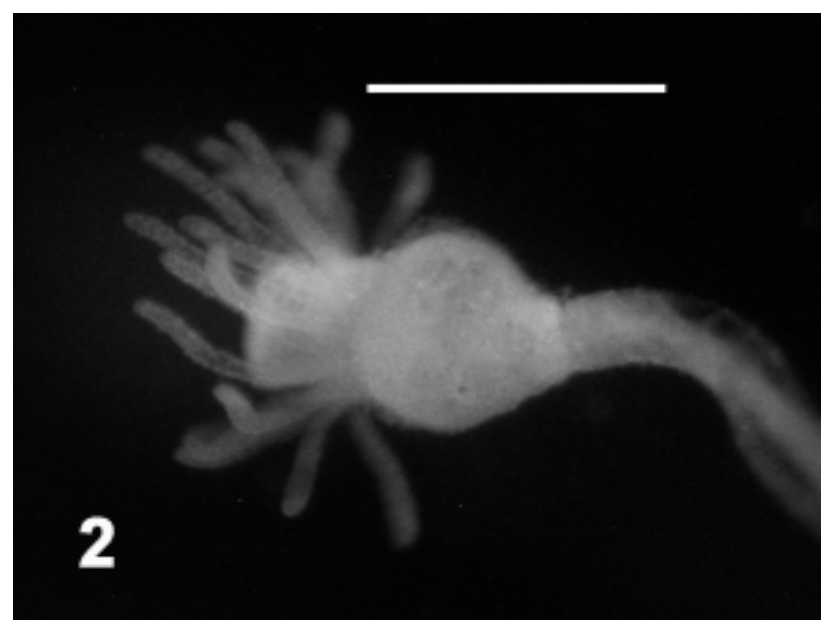

Figura 2. Hidrante de Eudendrium caraiuru. (escala: $500 \mu \mathrm{m}$ )

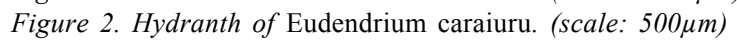

Eudendrium glomeratum; Marques 1993: 68-75, pl. 3; 2001: 361-369, figs. 5, 23-30; Migotto 1996: 122; Rosso \& Marques 1997: 417; Oliveira et al. 2000: 519-525, fig.1; Migotto et al. 2001: 289, 294-296; 2002: 12.

Eudendrium caraiuru Marques \& Oliveira, 2003:112, figs.1-19.

non Eudendrium glomeratum Picard, 1951: 338.

Material.- Trairi: praia de Mundaú, colônia sem gonóforo com hidrantes degenerados, 07.xi.2002, entremarés, 
col. A.C. Marques, álcool (MZUSP 513); praia de Fleixeiras, colônias sem gonóforo, 03.iii.2002, col. indeterminado, álcool (MZUSP 539); Pecém: praia de Pecém, colônias sem gonóforo, 24.x.2003, entremarés, col. A.C. Marques \& V. Shimabukuro, formol (MZUSP 597); Caucaia: praia do Pacheco, três amostras, colônias com gonóforo, 06.xi.2002, entremarés, col. A.C. Morandini \& A.C. Marques, álcool (MZUSP 509, 515 e 526); Cascavel: praia de Caponga, duas amostras, colônias sem gonóforo, 26.x.2003, entremarés, col. A.C. Marques \& V. Shimabukuro, formol (MZUSP 633 e 643).

Descrição.-Colônias eretas, com até $40 \mathrm{~mm}$ de altura, ramificadas, afasciculadas. Ramo principal (diâmetro: 142$348 \mu \mathrm{m}$ ) marrom escuro, surgindo de hidrorriza rastejante e ramificada. Ramificações até segunda ordem (133-161 $\mu \mathrm{m}$ em diâmetro), distribuídas quase alternadamente. Pedículos (diâmetro: 77-85 $\mu \mathrm{m}$ ) originam-se do hidrocaule ou de ramos de primeira e segunda ordens. Raros conjuntos de 3-8 anéis nos ramos. Hidrantes (diâmetro:192-354 $\mu \mathrm{m}$; comprimento: 380-530 $\mu \mathrm{m}$ ) com hipostômio em forma de urna, com 23-30 tentáculos (comprimento: 230-424 $\mu \mathrm{m}$ ) em verticilo único.

Gonóforos femininos com espádice simples; blastóstilos tendem a uma redução total de tentáculos e hipostômio.

Nematocistos de dois tipos: heterótrico euritelo microbásico $(6,59-6,76 \mu \mathrm{m} X 3,11-3,81 \mu \mathrm{m})$ nos tentáculos, hipostômio, corpo do hidrante e cenossarco; heterótrico euritelo mesobásico $(17,00-19,97 \mu \mathrm{m} X 8,00-11,70 \mu \mathrm{m})$ na base do hidrante, formando faixa ou em grumos, e no espádice do gonóforo feminino; nematocistos isolados sobre o hipostômio e no cenossarco.

Referências para o Brasil._ - todas as da lista sinonímica.

Ocorrências no Ceará. - Trairi, praias de Mundaú e Fleixeiras; Pecém, praia de Pecém; Caucaia, praia do Pacheco; Cascavel, praia de Caponga. Novo registro para o Ceará e para o litoral Nordeste.

Distribuição.- Brasil: São Paulo e Rio de Janeiro. Geral: endêmica para o Brasil.

Comentários. - Assim como as outras espécies do gênero Eudendrium, a identificação específica depende da determinação dos tipos de nematocistos. Caracteristicamente, a espécie pode contar com grumos (verrugas) de nematocistos grandes sobre o corpo do hidrante, cujo desenvolvimento varia, no entanto, desde uma faixa contínua até nematocistos esparsos.

Eudendrium caraiuru, assim como E. pocaruquarum, era conhecida apenas para a região Sudeste (Migotto et al. 2002). Suas colônias são relativamente menores e de aspecto mais delicado que as de E. carneum, o que pode estar relacionado a locais de menor hidrodinamismo. Essas espécies pertencem a um gênero cuja sistemática é considerada uma das mais confusas dentre os atecados (Marques 2001), com descrições e identificações antigas, dúbias ou falsas, e com a história taxonômica provavelmente relacionada a espécies crípticas. Por exemplo, E. caraiuru apenas recentemente foi reconhecida como distinta de E. glomeratum, por meio de estudos morfométricos, morfológicos e ecológicos que evidenciaram que as populações encontradas no Brasil divergiam da bem estabelecida linhagem mediterrânea (Marques \& Oliveira 2003).

\section{Eudendrium carneum Clarke, 1882}

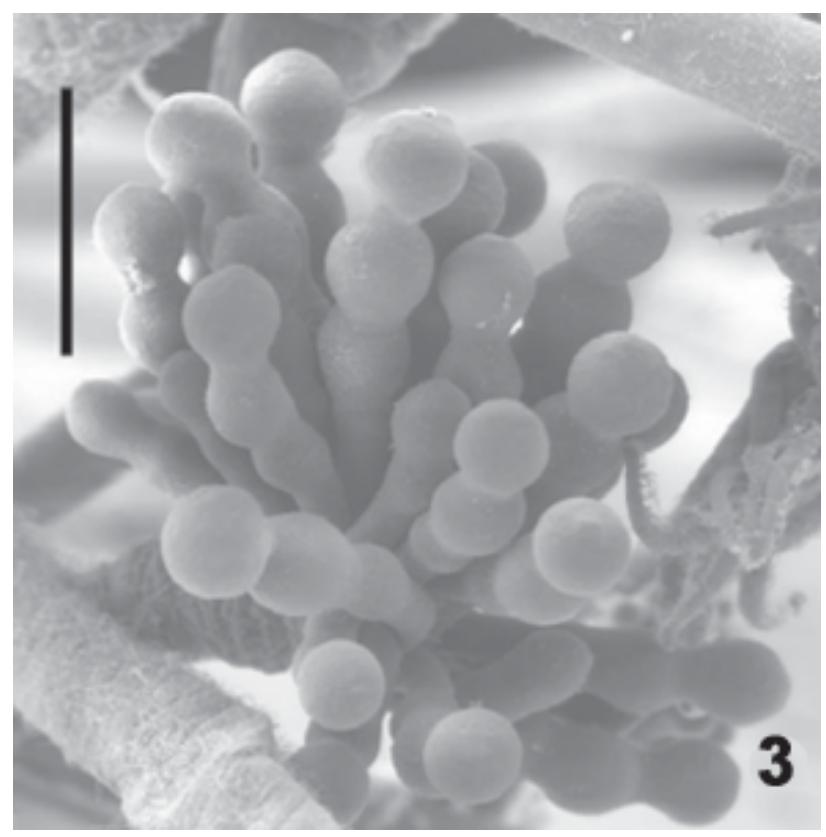

Figura 3. Microscopia eletrônica de varredura do gonozoóide masculino de Eudendrium carneum. (escala: $450 \mu \mathrm{m}$ )

Figure 3. Scanning electron micrograph of male gonozooid of Eudendrium carneum. (scale: $450 \mu \mathrm{m}$ )

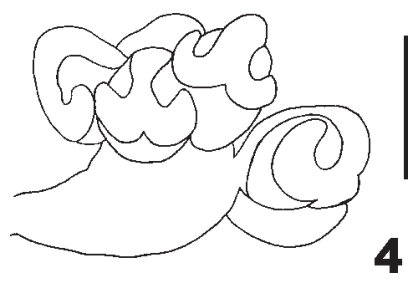

Figura 4. Gonozoóide feminino de Eudendrium carneum em desenvolvimento. (escala: $320 \mu \mathrm{m}$ )

Figure 4. Developing female gonozooid of Eudendrium carneum. (scale: $320 \mu \mathrm{m}$ ) 


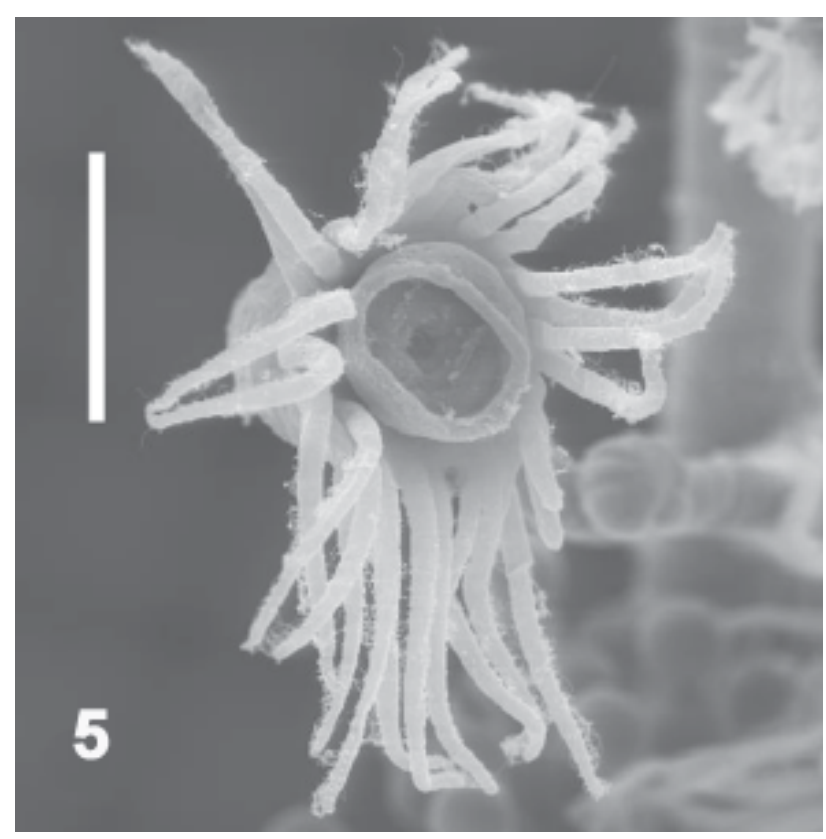

Figura 5. Microscopia eletrônica de varredura do hidrante de Eudendrium carneum. (escala: $600 \mu \mathrm{m}$ )

Figure 5. Scanning electron micrograph of the hydranth of Eudendrium carneum. (scale: $600 \mu \mathrm{m}$ )

Eudendrium carneum Clarke, 1882: 13; Vannucci 1954: 101-104, pl.1, fig. 1-9, pl. 2, fig. 8, pl. 4, fig. 2-5; Calder 1988: 43; Haddad 1992: 46 et seq.; Pires et al. 1992: 3; Marques 1993: 60-67, pl.2; Grohmann et al. 1997: 230; Nogueira et al. 1997: 367-368; Rosso \& Marques 1997: 417-420; Calder \& Maÿal 1998: 73; Kelmo \& de Santa-Isabel 1998: 63, 67-68, fig. 8; Oliveira et al. 2000: 519-526; Marques et al. 2000b: 9092, figs. 39-40; Marques 2001: 350-361, figs. 14-22; Migotto et al. 2002: 12; Grohmann et al. 2003: 8, 13, 15, 18 .

[Sinonímia mais completa em Marques 2001]

Material.- Itapipoca: praia da Baleia, colônias com gonóforos, 02.iv.1996, $15 \mathrm{~m}$, sobre recife artificial, col. W. Franklin Jr., álcool (MZUSP 589); Trairi: praia de Fleixeiras, duas amostras, colônias com gonóforos, 03.xi.2002, entremarés, col. A.C. Marques, A.C. Morandini \& S. Rabay, álcool (MZUSP 504 e 659); praia de Guajiru, colônias com gonóforos, 20.iii.2003, col. S. Rabay, álcool (MZUSP 680); Paracuru: praia de Paracuru, colônia com gonóforos femininos, 16.vi.2003, entremarés, col. indeterminado, formol (MZUSP 661); colônia sem gonóforos, 27.x.2003, entremarés, col. A.C. Marques \& V. Shimabukuro, formol (MZUSP 647); Pecém: praia de Pecém, sete amostras, colônias com e sem gonóforos, 24.x.2003, entremarés, col. A.C. Marques \& V. Shimabukuro, formol (MZUSP 538, 592, 595, 596, 598, 599 e 601); Caucaia: praia do Pacheco, colônias com gonóforos, 09.vii.2002, col. I. Martins, álcool; Fortaleza: praia de Mucuripe, colônias com gonóforos, 22.iv.1995, 15 m, sobre recife artificial, col. W. Franklin Jr., álcool (MZUSP 550);

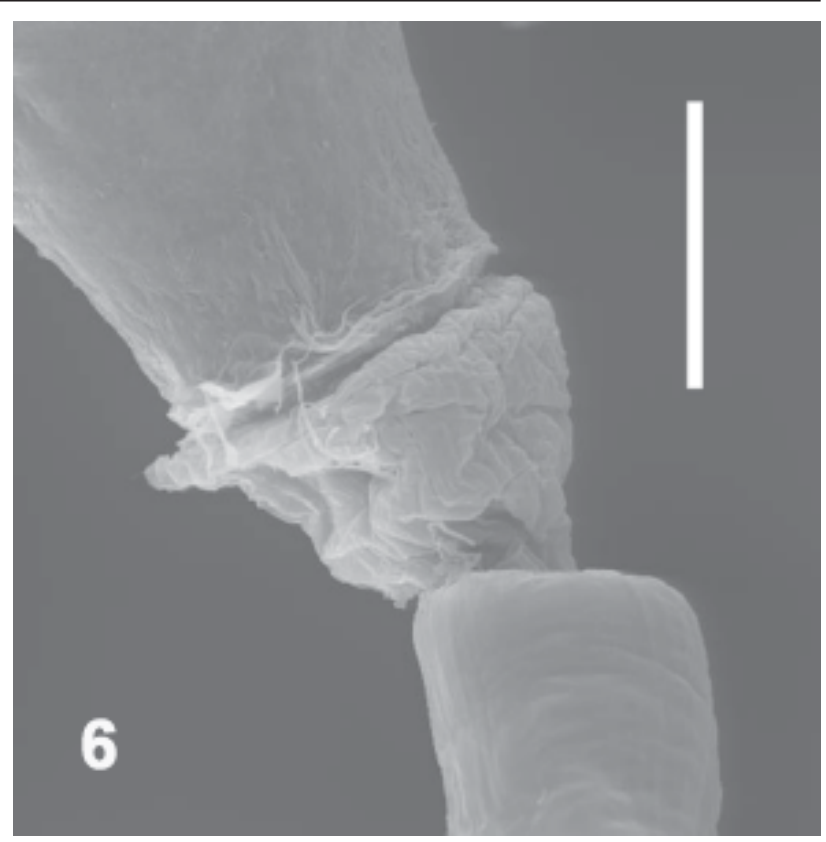

Figura 6. Microscopia eletrônica de varredura do sulco situado na base do hidrante de Eudendrium carneum. (escala: $300 \mu \mathrm{m}$ )

Figure 6. Scanning electron micrograph of the groove at the base of the hydranth of Eudendrium carneum. (scale: $300 \mu \mathrm{m}$ )

Cascavel: praia de Caponga, cinco amostras, colônias com e sem gonóforos, 26.x.2003, entremarés, col. A.C. Marques \& V. Shimabukuro, formol (MZUSP 610, 618, 619, 631 e 682).

Descrição.- Colônias eretas e ramificadas, fasciculadas na base, com até $80 \mathrm{~mm}$ de altura e ramificações radiais de até terceira ordem; ramificação tendendo a um único plano em colônias menores. Hidrorriza ramificada; hidrocaules (diâmetro: 225-259 $\mu \mathrm{m}$ ) e ramos secundários (diâmetro: 130-190 $\mu \mathrm{m}$ ) com perissarco liso e conjuntos espaçados de anelações (2-5). Pedículo (comprimento: 158$162 \mu \mathrm{m}$ ) surgindo das ramificações, enrugados ou totalmente anelados. Hidrantes (comprimento: 570-652 $\mu \mathrm{m}$; diâmetro: 274-332 $\mu \mathrm{m}$ ) com hipostômio em forma de trompete, cercado por verticilo de 18-25 tentáculos filiformes (comprimento: 531-588 $\mu \mathrm{m}$ ) e sulco conspícuo transversal basal no corpo.

Gonóforos fixos surgindo diretamente do corpo do hidrante, blastóstilos masculinos apresentando 12-14 esporossacos com 2-5 câmaras; femininos com espádices bifurcados e curvos sobre cada ovócito (5-7 ovócitos no total), blastóstilos com ovócitos maduros envolvidos por perissarco, formando cápsulas dispostas em cacho, cada uma com 2 fenestras não espessadas.

Referências para o Brasil._ Vannucci 1954: 101-104, pl.1, fig. 1-9, pl. 2, fig. 8, pl. 4, fig. 2-5; Haddad 1992: 46 et seq.; Pires et al. 1992: 3; Marques 1993: 60-67, pl.2; Grohmann et al. 1997: 230; Nogueira et al. 1997: 367, 368; Rosso \& 
Marques 1997: 417, 420; Calder \& Maÿal 1998: 72-74, 77; Kelmo \& de Santa-Isabel 1998: 63, 67-68, fig. 8; Oliveira et al. 2000: 519-526; Marques 2001: 350-361, figs. 14-22; Migotto et al. 2002: 12; Grohmann et al. 2003: 6, 8, 13, 15, 18; Marques \& Migotto 2004: 174.

Ocorrências no Ceará.- Itapipoca, praia da Baleia; Trairi, praia de Fleixeiras; Paracuru, praia de Paracuru; Taíba, praia de Taíba, Pecém, praia de Pecém; Fortaleza, praia de Mucuripe; Cascavel, praia de Caponga. Novo registro para o Ceará.

Distribuição.- Brasil: Santa Catarina à Bahia, Pernambuco e Fernando de Noronha. Geral: cosmopolita de águas tropicais e subtropicais.

Comentários.- A espécie Eudendrium carneum foi registrada para o Brasil por Vannucci (1954), a partir de material proveniente de Ilhabela, Santos e Cananéia (SP). Além de eurihalina e euritérmica, fato que explica a ampla distribuição na costa brasileira, como notado por Vannucci (1954), a espécie é também euribática, visto que já foi registrada para profundidades de até $70 \mathrm{~m}$ na plataforma continental brasileira, em região próxima ao estado do Espírito Santo (Grohmann et al. 2003). No Nordeste, a espécie foi registrada para a região estuarina de Pernambuco, sendo umas das espécies de Hydrozoa mais freqüentes, ocorrendo em locais que iam desde a zona marinha até o interior do estuário (Calder \& Maÿal 1998). É também reportada no norte do estado da Bahia, onde é a espécie mais abundante da região (Kelmo \& de Santa-Isabel 1998), e para Fernando de Noronha (Pires et al. 1992). Essa ampla distribuição pode também estar relacionada a aspectos morfológicos, uma vez que forma colônias robustas e de grande porte.

O material estudado concorda com a descrição detalhada de espécimes da região Sudeste (Vannucci 1954, Marques 2001). Diferencia-se das outras espécies brasileiras de Eudendrium por apresentar o espádice feminino bifurcado.

\section{Eudendrium pocaruquarum Marques, 1995}

Eudendrium pocaruquarum Marques, 1995: 35-40, fig. 1-9; Rosso \& Marques 1997: 417; Marques 2001: 374375, figs. 3-4; Migotto et al. 2002: 12.

Material.- Pecém: praia de Pecém, duas amostras, colônias sem gonóforos, 24.x.2003, entremarés, col. A.C. Marques \& V. Shimabukuro, formol (MZUSP 509 e 602); Caucaia: praia do Pacheco, colônia sem gonóforo, 06.xi.2002, entremarés, col. A.C. Marques, álcool (MZUSP 549).
Descrição.-Colônias eretas, ramificadas até segunda ordem, afasciculadas, ca. $15 \mathrm{~mm}$ de altura. Ramo principal surgindo de hidrorriza lisa e ramificada; ramo principal (diâmetro: 120-126 $\mu \mathrm{m}$ ) e secundários (diâmetro: 111-125 $\mu \mathrm{m}$ ) com 2-8 anelações no perissarco. Pedículos (comprimentos: 1180-2816 $\mu \mathrm{m}$; diâmetros: 126-170 $\mu \mathrm{m}$ ) enrugados, surgindo dos ramos principal e secundários. Hidrantes terminais (comprimento: $325-500 \mu \mathrm{m}$ ), com verticilo de 18-21 tentáculos filiformes (diâmetro: 242-265 $\mu \mathrm{m}$; comprimento: 430-455 $\mu \mathrm{m}$ ) ao redor de hipostômio em forma de trompete; sulco transversal na porção basal do corpo.

Gonóforos ausentes.

Nematocistos heterótricos microbásicos euritelos grandes (13-14 $\mu \mathrm{m} X$ 5,72-7 $\mu \mathrm{m})$ e pequenos (5,8-7,3 $\mu \mathrm{m} \mathrm{X}$ 3,11-3,81 $\mu \mathrm{m}$ ) distribuídos no corpo do hidrante e no hipostômio; apenas euritelos pequenos nos tentáculos.

Referências para o Brasil. — todas as da lista sinonímica.

Ocorrências no Ceará.- Pecém, praia de Pecém; Caucaia, praia do Pacheco. Novo registro para o Ceará e para o litoral nordeste.

Distribuição.- Brasil: São Paulo. Geral: endêmica para o Brasil.

Comentários.- O material concorda com descrição de material encontrado na região Sudeste (Marques 1995), apenas diferindo na forma da colônia, que é mais ramificada no material do Sudeste.

\section{Eudendrium sp.}

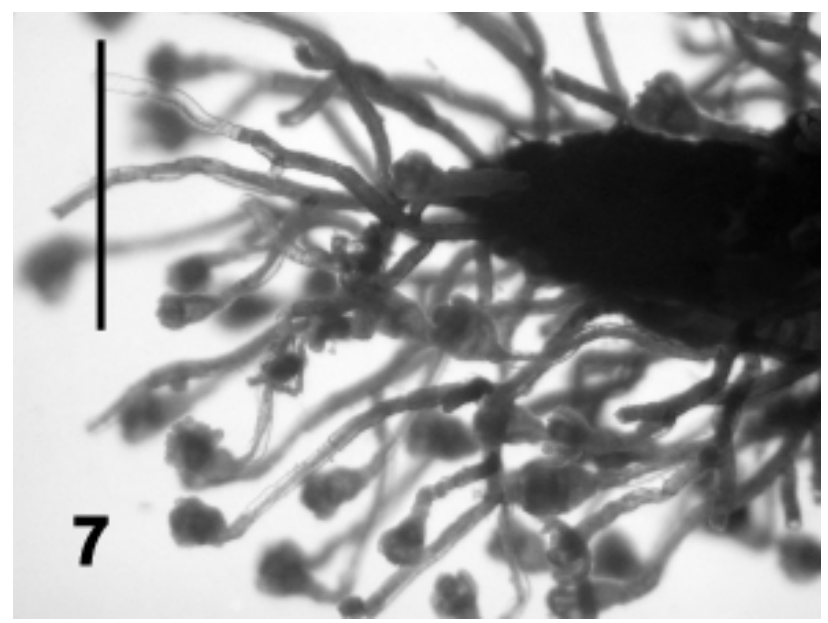

Figura 7. Colônia de Eudendrium sp. sobre alga. (escala: 2mm) Figure 7. Colony of Eudendrium sp. on algae. (scale: 2mm) 
Material - Cascavel: praia Caponga, colônias sem gonóforos, 26.x.2003, entremarés e sobre alga, col. A.C. Marques \& V. Shimabukuro, formol (MZUSP 615).

Descrição.-Colônias estoloniais, afasciculadas, com ramificações de até segunda ordem. Hidrorriza ramificada, dando origem a hidrocaule e ramos secundários indistintos (diâmetro: 97-139 $\mu \mathrm{m}$ ); hidrocaules com perissarco liso e conjuntos de anelações conspícuas apenas na base das ramificações. Pedículo surgindo das ramificações, indistinto do hidrocaule, pouco enrugado ou totalmente liso. Hidrantes (comprimento: 481-796 $\mu \mathrm{m}$; diâmetro na base dos tentáculos: 216-405 $\mu \mathrm{m}$ ) com hipostômio em forma de trompete, cercado por verticilo de 14-17 tentáculos filiformes, contraídos após preservação.

Gonóforos ausentes.

Nematocistos heterótricos anisorrizas (18,58-19,8 $\mu \mathrm{m}$ $\mathrm{X} 8,25-9,57 \mu \mathrm{m})$ e heterótricos microbásicos euritelos pequenos (7,71-8,71 $\mu \mathrm{m} X 3,36-3,97 \mu \mathrm{m})$ distribuídos no corpo do hidrante e no hipostômio; apenas euritelos pequenos nos tentáculos.

Comentários. - O material apresenta internamente inclusões semelhantes a zooxantelas (diâmetro: 5,9-9,0 $\mu \mathrm{m}$ ) no corpo do hidrante. Estruturas semelhantes foram reportadas apenas em outra espécie do gênero, Eudendrium moulouyensis Marques, Peña Cantero \& Vervoort, 2000c, com a qual, no entanto, Eudendrium sp. apresenta diferenças no cnidoma. Uma vez que as estruturas reprodutivas estão ausentes no material examinado, é ainda prematuro descrevê-lo como uma nova espécie.

\section{Ordem Capitata Kuhn, 1913 \\ Família Corynidae Johnston, 1836 \\ Coryne sp.}

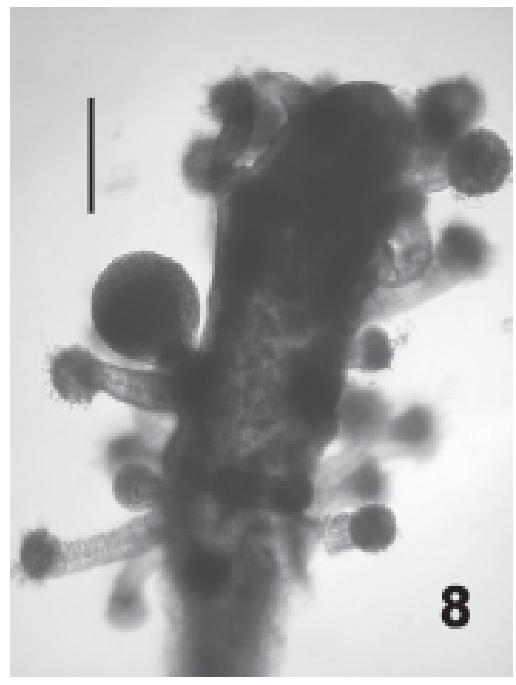

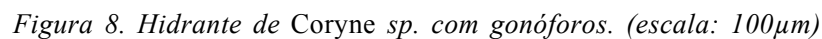
Figure 8. Hydranth of Coryne sp. with gonophores. (scale: 10 $\mu$ im)
Material. - Trairi: praia do Mundaú, colônia com gonóforo, 04.xi.2002, entremarés, sobre alga, col. A.C. Marques, álcool (MZUSP 535).

Descrição.-Colônia estolonial, afasciculada, surgindo de hidrorriza rastejante (diâmetro: 145-170 $\mu \mathrm{m}$ ) com anelações menos evidentes, quase lisa. Pedículos (comprimento: 828,685535,32 $\mu \mathrm{m}$; diâmetro: 134-170 $\mu \mathrm{m}$ ) surgindo diretamente da hidrorriza, com anéis conspícuos próximos à base dos hidrantes. Hidrantes em forma de clava (comprimento: 760$1460 \mu \mathrm{m}$; diâmetro máximo: 304-376 $\mu \mathrm{m}$ ), com 20-28 tentáculos capitados (comprimento: 148,74-427,22 $\mu \mathrm{m}$ ), irregularmente distribuídos ou em 4-5 verticilos alternados.

Gonóforos, na forma de esporossacos fixos, esféricos (diâmetro: 134,11-278,55 $\mu \mathrm{m}$ ), surgindo entre tentáculos, no corpo do hidrante.

Ocorrências no Ceará.- Trairi, praia do Mundaú. Novo registro do gênero para o Ceará e para o litoral nordeste.

Comentários. - A única espécie do gênero Coryne descrita para o Brasil é C. pusilla Gaertner, 1774, encontrada nas regiões Sul e Sudeste. Os trabalhos que citam a espécie (Haddad 1992, Grohmann et al. 1997, Nogueira et al. 1997, como identificação incerta), no entanto, não apresentam descrições ou figuras. A espécie foi coletada sobre alga.

Família Pennariidae McCrady, 1859

Pennaria disticha Goldfuss, 1820

Pennaria disticha Goldfuss, 1820: 89; Gibbons \& Ryland 1989: 387, fig. 5A-C; Migotto 1996: 25; Calder \& Maÿal 1998: 73; Migotto et al. 2002: 10, fig. 4; Grohmann et al. 2003: 13, 15, 18.

Halocordyle fragilis Vannucci, 1951a: 76-77, pl. 1, figs. 2-3; 1951b: 106-115.

Halocordyle disticha; Millard 1975: 41-42, fig.16C-G; Migotto \& Silveira 1987: 96-99, figs. 1-2; Calder 1988: 56-60, figs. 43a-b, 44a-b, 45a-h; Silveira \& Migotto 1991: 437-442, fig. 1A-B; Pires et al. 1992: 4; Grohmann et al. 1997: 230; Rosso \& Marques 1997: 417, 420; Kelmo \& de Santa-Isabel 1998: 63, 68-69, fig. 9.

[Sinonímia mais completa em Calder 1988]

Material.- Trairi: praia de Fleixeiras, colônias sem gonóforos, 03.xi.2002, entremarés, col. A.C. Marques, álcool (MZUSP 534); Caucaia: praia do Pacheco, colônias com hidrantes degenerados, 9.vii.2002, col. I. Martins, álcool (MZUSP 525); Fortaleza: praia de Sabiaguaba, colônias com gonóforos, 05.vi.1993, sobre rocha, col. H. Matthews, álcool (MZUSP 558); Cascavel: praia de Caponga, colônias com

http://www.biotaneotropica.org.br 
gonóforos e hidrantes degenerados, 17.v.1998, sobre esponja e ascídia, col. R. Aquino \& A. Pereira, álcool (MZUSP 503).

Descrição.- Colônias eretas, monopodiais e afasciculadas, com até $50 \mathrm{~mm}$ de altura, surgindo de hidrorriza ramificada e lisa. Ramificação geralmente até segunda ordem; ramos alternados, levemente curvos e com hidrante terminal. Hidrocaule do ramo principal (diâmetro: 252-340 $\mu \mathrm{m}$ ) de coloração marrom escuro; hidrocládios (diâmetro:165-180 $\mu \mathrm{m}$ ) mais claros que o ramo principal, ambos com conjuntos de 3-7 anéis intercalados por porções lisas. Pedículos surgindo principalmente do lado adaxial dos ramos, com anelações na base. Hidrante (comprimento: 458-595 $\mu \mathrm{m}$, diâmetro máximo: $234-330 \mu \mathrm{m}$ ) em forma de clava, com hipostômio cônico cercado por 6-8 tentáculos capitados curtos (comprimento: 95-115 $\mu \mathrm{m}$ ) e com uma coroa de 10-12 tentáculos filiformes longos (comprimento: 345-520 $\mu \mathrm{m}$ ) na região aboral.

Gonóforos ovais ou piriformes, com canais radiais, surgindo diretamente no corpo do hidrante, entre os tentáculos filiformes e capitados.

Referências para o Brasil._- Vannucci 1951a: 76-77, pl. 1, figs. 2-3; 1951b: 106-115; Migotto \& Silveira 1987: 9699, figs. 1-2; Silveira \& Migotto 1991: 437-442, fig. 1A-B; Pires et al. 1992: 4; Migotto 1996: 25; Grohmann et al. 1997: 230; Rosso \& Marques 1997: 417, 420; Calder \& Maÿal 1998: 73; Kelmo \& de Santa-Isabel 1998: 63, 68-69, fig. 9; Migotto et al. 2002: 10, fig. 4; Grohmann et al. 2003: 8, 13, 15, 18; Marques \& Migotto 2004: 174.

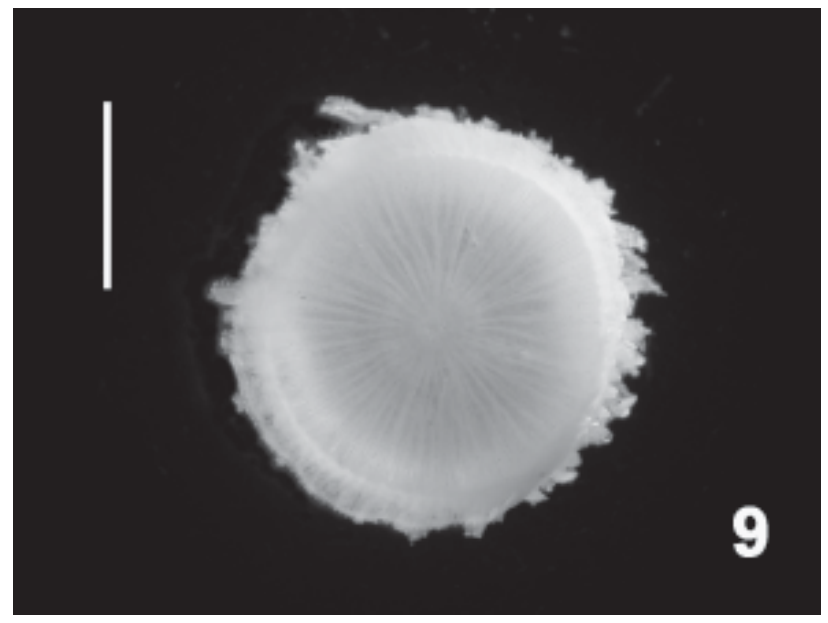

Figura 9. Vista aboral de uma colônia planctônica de Porpita porpita. (escala: $5 \mathrm{~mm}$ )

Figure 9. Aboral view of a planktonic colony of Porpita porpita. (scale: $5 \mathrm{~mm}$ )
Ocorrências no Ceará. - Trairi, praia de Fleixeiras; Caucaia, praia do Pacheco; Fortaleza, praia de Sabiaguaba; Cascavel, praia de Caponga. Novo registro para o Ceará.

Distribuição.- Brasil: Santa Catarina à Bahia, Pernambuco, Fernando de Noronha. Geral: cosmopolita (oceanos Atlântico, Índico e Pacífico).

Comentários. - O material cearense assemelha-se àquele descrito para o litoral paulista (Vannucci 1951a, Migotto \& Silveira 1987) e baiano (Kelmo \& de Santa-Isabel 1998), exceto com relação à altura dos hidrantes que é bem menor (595 $\mu \mathrm{m}$ vs. $1600 \mu \mathrm{m})$. Não há, tampouco, grandes diferenças com relação às descrições de materiais do sul da África (Millard 1975), das Bermudas (Calder 1988) e das Ilhas Fiji (Gibbons \& Ryland 1989), exceto no número dos tentáculos orais capitados, maior nos espécimes de Fiji e sul da África.

No Ceará, Pennaria disticha ocorre na zona entremarés, sobre rocha, ascídias e algas. A espécie apresenta alta plasticidade fenotípica (Silveira \& Migotto 1991) como resposta a variações no hidrodinamismo. A forma encontrada na maioria dos locais no litoral cearense é a penada, presumida como associada a locais com correntes unidirecionais.

Família Porpitidae Goldfuss, 1818

Porpita porpita (Linnaeus, 1758)

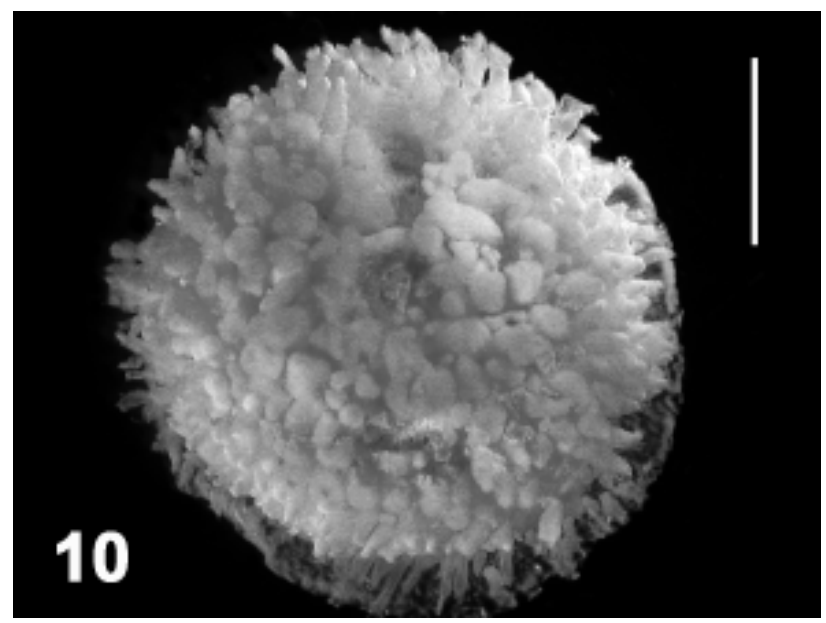

Figura 10. Vista oral de uma colônia planctônica de Porpita porpita. (escala: $5 \mathrm{~mm}$ )

Figure 10. Oral view of a planktonic colony of Porpita porpita. (scale: $5 \mathrm{~mm}$ )

http://www.biotaneotropica.org.br 
Medusa porpita Linnaeus, 1758: 659.

Porpita porpita; Schneider, 1898: 194.

[Sinonímia mais completa em Calder 1988]

Material - Beberibe: praia do Morro Branco, 28.vi.2001, col. E. Ximenes, álcool (MZUSP 562).

Descrição.- colônias polimórficas do plêuston; flutuador quase circular, com superfície superior levemente convexa e raios relacionados aos canais gastrodérmicos. Superfície inferior do flutuador com um gastrozoóide central curto, com boca terminal, circundado por gonozoóides em forma de clava com verrugas de nematocistos, e dactilozoóides com tentáculos capitados distais, sem boca.

Referências para o Brasil.-Alvariño 1981: 435, fig. 174-88; Kelmo \& de Santa-Isabel 1998: 70, fig. 11; Migotto et al. 2002: 10 .

Ocorrências no Ceará.- Beberibe, praia de Morro Branco. Novo registro para o Ceará.

Distribuição.- Brasil: São Paulo e Bahia. Geral: cosmopolita de águas tropicais e temperadas.

Comentários.- espécie pleustônica e oceânica, ocasionalmente encontrada em grandes quantidades encalhada nas praias. Diferencia-se de outra espécie da família que ocorre no Brasil (Velella velella) pela forma circular da colônia e ausência de uma vela perpendicular ao flutuador.

Família Sphaerocorynidae Prevót, 1959

?Sphaerocoryne sp.

Material - Trairi: praia de Fleixeiras, colônias sem gonóforos, 03.xi.2002, entremarés, col. A.C. Marques, álcool (MZUSP 520).

Descrição.- colônia estolonial com hidrocaule afasciculado diminuto e hidrante terminal. Perissarco do hidrocaule tênue e liso. Hidrantes piriformes com região mediana dilatada. Hipostômio cônico, sua base (equivalente à região expandida do hidrante) circundada por tentáculos capitados dispostos irregularmente, formando uma faixa.

Ocorrências no Ceará.- Trairi, praia de Fleixeiras. Novo registro do gênero para o Ceará e para o litoral nordeste.
Comentários. - há três registros do gênero Sphaerocoryne para o Brasil: Sphaerocoryne arcuata (Haeckel, 1879) por Vannucci (1951b) (sem localidade específica), Sphaerocoryne sp. e Sphaerocoryne bedoti para litoral capixaba por Grohmann et al. (1997 e 2003, respectivamente). O material examinado é representado por alguns poucos hidrantes sem gonóforos, mal preservados. Embora sua identificação seja incerta, não descartamos a possibilidade de tratar-se de Sphaerocoryne bedoti Pictet, 1893, espécie registrada para o Caribe, Colômbia (Mergner \& Wedler 1977) e Bermudas (Calder 1988). O espécime foi encontrado sobre o sertulariídeo Sertularella cylindritheca, na zona entremarés.

\section{Agradecimentos}

Os autores agradecem a Helena Matthews Cascon (Universidade Federal do Ceara - UFC), Tito Lotufo (UFC), André C. Morandini (Universidade de São Paulo - USP), Marcelo O. Soares (UFC), Soraya Rabay (UFC) e ao Laboratório de Malacologia da UFC pelo apoio durante as coletas, assim como aos técnicos Enio Mattos e Eduardo Mattos do Departamento de Zoologia da USP pelo auxílio na microscopia eletrônica. Os materiais foram obtidos principalmente através do PROBIO ("Projeto de conservação e utilização sustentável da diversidade biológica brasileira") do Ministério do Meio Ambiente. VS teve bolsa do CNPq e ACM teve auxílios FAPESP2001/02626-7, 2003/02432-3 e 2004/09961-4.ACM e AEM têm bolsa de pesquisa e auxílios do CNPq.

\section{Referências bibliográficas}

ALVARIÑO, A. 1981. Siphonophorae. In Atlas del zooplancton del Atlántico sudoccidental y metodos de trabajo con el zooplancton marino (D. Boltovskoy, ed.). INIDEP, Mar del Plata, p.383-441.

CALDER, D.R. 1988. Shallow-water hydroids of Bermuda: The Athecatae. Life Sci. Contri. R. Ontario Mus. 148: 1-107.

CALDER, D.R. \& MAÿAL, E.M. 1998. Dry season distribution of hydroids in a small tropical estuary, Pernambuco, Brazil. Zool. Verh. Leiden 323: 69-78.

CLARKE, S.F. 1882. New and interesting hydroids from Chesapeake Bay. Mem. Boston Soc. Nat. His. 3(4):135142.

GAERTNER, J. 1774. In Pallas, P.S., Spicilega zoologica quibus novae imprimus et obscurae animalium species. Fasc. 10. Berolini, August Lange. 41p.

GIBBONS, M.J. \& RYLAND, J.S. 1989. Intertidal and shallow water hydroids from Fiji. I. Athecata to Sertulariidae. Mem. Qd Mus. 27(2): 377-432.

GOLDFUSS, G.A. 1820. Handbuch der Zoologie. I. Abtheilung. Nürnberg, Johann Leonhard Schrag. 696p. 
GROHMANN, P.A., SOUZA, M.M. \& NOGUEIRA, C.C. 1997. Hydroids from the vicinity of a large industrial area in Vitória, Espírito Santo, Brazil. Proceedings of the VI International Conference on Coelenterate Biology, Leiden, The Netherlands: 227-232.

GROHMANN, P.A., NOGUEIRA, C.C. \& da SILVA, V.M.A.P. 2003. Hydroids (Cnidaria, Hydrozoa) collected on the continental shelf of Brazil during the Geomar X Oceanographic Operation. Zootaxa. 299: 1-19.

HADDAD, M.A. 1992. Hidróides (Cnidaria, Hydrozoa) de costões rochosos do litoral sul do Estado do Paraná. Tese de Doutorado, Universidade de São Paulo, São Paulo.

HAECKEL, E. 1879. Das System der Medusen. Erster Theil einer Monographie der Medusen. Denkschriften der Medicinisch-Naturwissenschaftlichen Gesellschaft zu Jena $1.360 p$.

KELMO, F. \& DE SANTA-ISABEL, L.M. 1998. The athecatae hydroids (Cnidaria, Hydrozoa) from Northern Bahia, Brazil. Rev. Biol. Trop. 46 (supl.5): 61-72.

LINNAEUS, C. 1758. Systema naturae per regna tria naturae, secundum classes, ordines, genera, species cum characteribus, differentiis, synonymis, locis. Editio decima, reformata. Holmiae (Stockholm), Laurentii Salvii. 823p.

MARISCAL, R.N. 1974. Chapter 3. Nematocysts. In Coelenterate Biology (L. Muscatine \& H.M. Lenhoff, eds.). Academic Press, New York, p.129-178.

MARQUES, A.C. 1993. Sistemática dos Eudendriidae L. Agassiz, 1862 (Cnidaria, Hydrozoa) do litoral Paulista. Dissertação de Mestrado, Universidade de São Paulo, São Paulo.

MARQUES, A.C. 1995. Eudendrium pocaruquarum n. sp. (Hydrozoa, Eudendriidae) from the southeastern coast of Brazil, with remarks on taxonomic approaches to the family Eudendriidae. Contr. Zool. 65 (1): 35-40.

MARQUES, A.C. 2001. O gênero Eudendrium (Hydrozoa, Anthomedusae, Eudendriidae) no Brasil. Pap. Av. Zool. 41(22): 329-405.

MARQUES, A.C \& COLLINS, A.G. 2004. Cladistic analysis of Medusozoa and cnidarian evolution. Invert. Biol. 123(1):23-42.

MARQUES, A.C. \& MIGOTTO, A.E. 2004. Hidrozoários (Cnidaria) marinhos bentônicos da Estação Ecológica Juréia-Itatins. In Ambiente, Flora e Fauna da Estação Ecológica Juréia-Itatins (Marques, O.A.V. \& Duleba, W. eds.). Holos, Ribeirão Preto, p.170-176.

MARQUES, A.C. \& OLIVEIRA, O.M.P. 2003. Eudendrium caraiuru sp. n. (Hydrozoa; Anthoathecata; Eudendriidae) from the southeastern coast of Brazil. Zootaxa. 307: 1-12.
MARQUES, A.C., MERGNER, H., HÖINGHAUS, R. \& VERVOORT, W. 2000a. Bimeria vestita (Hydrozoa: Anthomedusae: Bougainvilliidae) senior synonym of Eudendrium vestitum (Hydrozoa: Anthomedusae: Eudendriidae). Zool. Med. Leiden. 73 (22): 321-325.

MARQUES, A.C., MERGNER, H., HÖINGHAUS, R., SANTOS, C.M.D. \& VERVOORT, W. 2000b. Morphological study and taxonomical notes on Eudendriidae (Cnidaria; Hydrozoa; Athecatae/ Anthomedusae). Zool. Med. Leiden. 74(5): 75-118.

MARQUES, A.C., PEÑA CANTERO, A.L. \& VERVOORT, W. 2000c. Mediterranean species of Eudendrium Ehrenberg, 1834 (Hydrozoa, Anthomedusae, Eudendriidae) with the description of a new species. J. Zool. 252: 197-213.

MARQUES, A.C., MORANDINI, A.C. \& MIGOTTO, A.E. 2003. Synopsis of knowledge on Cnidaria Medusozoa from Brazil. Biota Neotropica, 3 (2):1-18. http:// www.biotaneotropica.org.br/v3 n $2 / \mathrm{pt} /$ abstract?inventory+BN01203022003

MERGNER, H. \& WEDLER, E. 1977. Ueber die Hydropolypenfauna des Roten Meeres und seiner Ausgänge. "Meteor" Forschungs-Ergebnisse. (D)24:1-32.

MigOTTO, A.E. 1996. Benthic shallow water hydroids (Cnidaria, Hydrozoa) of the coast of São Sebastião, Brasil, including a check list of the Brazillian hydroids. Zool.Verh. Leiden 306: 1-125.

MIGOTTO, A.E. \& SILVEIRA, F.L. 1987. Hidróides (Cnidaria, Hydrozoa) do litoral sudeste e sul do Brasil: Halocordylidae, Tubulariidae e Corymorphidae. Iheringia. Sér. Zool. 66: 95-115.

MigotTO, A.E. \& MARQUES, A.C. 1999. Hydroid and medusa stages of the new species Ectopleura obypa (Cnidaria: Hydrozoa: Tubulariidae) from Brazil. Proc. biol. Soc. Wash. 112(2): 303-312.

MIGOTTO, A.E. \& MARQUES, A.C. 2006. Invertebrados Marinhos. In Avaliação do Estado do Conhecimento da Biodiversidade Brasileira (T. Lewinsohn, org.). Ministério do Meio Ambiente, Brasília, p.149-202.

MIGOTTO, A.E., MARQUES, A.C. \& FLYNN, M.N. 2001. Seasonal recruitment of hydroids (Cnidaria) on experimental panels in the São Sebastião Channel, Southeastern Brazil. Bull. Mar. Sci. 68(2): 287-298.

MIGOTTO, A.E., MARQUES, A.C., MORANDINI, A.C. \& SILVEIRA, F.L. 2002. Checklist of the Cnidaria Medusozoa of Brazil. Biota Neotropica 2(1):1-31. http:// www.biotaneotropica.org.br/v2n $1 / \mathrm{pt} /$ fullpaper?bn01102012002+en

MILLARD, N.A.H. 1975. Monograph on the Hydroida of Southern Africa. Ann. S. Afr. Mus. 68: 1-513. 
NOGUEIRA, C.C., GROHMANN, P.A. \& DASILVA, V.M.A.P. 1997. Hydroids from the vicinity of a nuclear power plant site (CNAAA-Unidade I) at Angra-dos-Reis, Rio de Janeiro, southeastern Brazil. Proceedings of the VI International Conference on Coelenterate Biology, Leiden, The Netherlands: 365-369.

OLIVEIRA, O.M.P., MARQUES, A.C. \& MIGOTTO, A.E. 2000. Morphometric patterns of two fouling Eudendrium spp. (Hydrozoa, Anthomedusae, Eudendriidae) from São Sebastião (SP, SE Brazil). Braz. Arch. Biol. Tech. 43(5): 519-526.

ÖSTMAN, C. 2000. A guideline to nematocyst nomenclature and classification, and some notes on the systematic value of nematocysts. Scientia Marina. 64(suppl.1): 31-46.

PICARD, J. 1951. Note sur les hydraires littoraux de Banyulssur-Mer. Vie et Milieu 2: 338-349.

PICTET, C. 1893. Étude sur les hydraires de la Baie d'Amboine. Revue Suisse de Zoologie 1: 1-64.

PIRES, D.O., CASTRO, C.B., MIGOTTO,A.E. \& MARQUES, A.C. 1992. Cnidários bentônicos do Arquipélago de Fernando de Noronha, Brasil. Bolm Mus. nac. Rio de Janeiro, Zool. 354: 1-21.

ROSSO, S. \& MARQUES, A.C. 1997. Is there any conspicuous geographical pattern in intertidal hydrozoan distribution along the coast of São Paulo State, Southeastern Brazil. Proceedings of the VI International Conference on Coelenterate Biology, Leiden, The Netherlands: 415-422.

SCHNEIDER, K.C. 1898. Hydropolypen von Rovigno, nebst Uebersicht über das System der Hydropolypen im Allgemeinen. Zoologische Jahrbücher, Abtheilung für Systematik, Geographie und Biologie der Thiere 10(4): 472-555.

SILVEIRA, F.L. \& MIGOTTO, A.E. 1991. The variation of Halocordyle disticha (Cnidaria, Athecata) from the Brazilian coast: an environmental indicator species? Hydrobiologia 216/217: 437-442.

VANNUCCI-MENDES, M. 1946. Hydroida Thecaphora do Brasil. Arq. Zool. Estado São Paulo 4: 535-538.

VANNUCCI, M. 1951a. Hydrozoa e Scyphozoa existentes no Instituto Paulista de Oceanografia. I. Bolm Inst. oceanogr., S Paulo 2(1): 67-98.

VANNUCCI, M. 1951b. Distribuição dos Hydrozoa até agora conhecidos nas costas do Brasil. Bolm Inst. oceanogr., S Paulo 2(1): 105-124.

VANNUCCI, M. 1954. Hydrozoa e Scyphozoa existentes no Instituto Oceanográfico. II. Bolm Inst. oceanogr., S Paulo 5(1-2): 95-149.

WEILL, R. 1934. Contribution à l'étude des cnidaires et de leurs nématocystes. I. Recherches sur les nématocystes (morphologie, physiologie, dévelopement). II. Le valeur taxonomique du cnidome. Trav. Stn zool. Wimereux, 11: 1-351.
WRIGHT, T.S. 1859. Observations on British zoophytes. Edinburgh New Philosophical Journal. 10: 105-114.

Título: Fauna de hidrozoários atecados (Hydrozoa, Anthoathecata) da costa do Estado do Ceará, Brasil.

Autores: Shimabukuro, V., Marques, A.C. and Migotto, A.E.

Biota Neotropica, Vol. 6 ( número 3 ): 2006

http://www.biotaneotropica.org.br/v6n3/pt/ abstract?inventory+bn00806032006

Recebido em 08/09/05 - Versão reformulada recebida em 08/06/2006 - Publicado em 01/09/06

ISSN 1676-0603 